\title{
Molecular wires: tuning of electron transport
}

\author{
Santanu K. Maiti ${ }^{1,2, *}$ \\ ${ }^{1}$ Theoretical Condensed Matter Physics Division, Saha Institute of Nuclear Physics, \\ 1/AF, Bidhannagar, Kolkata-700 064, India \\ ${ }^{2}$ Department of Physics, Narasinha Dutt College, 129, Belilious Road, Howrah-711 101, India
}

\begin{abstract}
Electron transport characteristics through molecular wires are studied by using the Green's function formalism. Parametric calculations are performed based on the tight-binding model to investigate the transport properties through the wires. The transport characteristics are significantly influenced by (a) the interference effects, (b) chemical substituent group, (c) molecule-to-electrode coupling strength and (d) the gate voltage, and, here we focus our results in these aspects. In this article we also discuss the noise power of current fluctuations. The noise power gives key information about the electron correlation which is obtained by calculating the Fano factor $(F)$ and the complete knowledge of the current fluctuations is very essential to fabricate efficient molecular devices.
\end{abstract}

PACS No.: 73.23.-b; 73.63.Rt; 73.40.Jn; 81.07.Nb

Keywords: Molecular wires; Interference effects; Conductance; $I-V$ characteristic; Shot noise.

${ }^{*}$ Corresponding Author: Santanu K. Maiti

Electronic mail: santanu.maiti@saha.ac.in 


\section{Introduction}

The present miniaturization has provided us to explore electron transport on the scale of a single molecule. The advantage of molecules as an electronic building blocks is that the molecules can be engineered to have some built in functionality, acting, for examples, as light sensitive switches, gates, or transport elements. Furthermore, molecules are very small and hence molecular systems could provide a way to down the scale of electronic devices even further, especially in self-assembly can be used to fabricate nanoscale circuits. Therefore, the field of molecular electronics is receiving increasing attention from fundamental scientists and industry alike. In 1974, Aviram and Ratner [1] first described electron transport through single molecule electronic device-the molecular rectifier. Later, the developments of nanoscience and technologies have provided several possible route for the construction and characterization of single-molecule devices 2, 2, 4, 5, 6, 7. From experimental developments, theory can give a better insight in understanding the new mechanism of conductance through molecules placed between two non-superconducting electrodes with few nanometer separation, yet the complete knowledge of the conduction mechanism in this scale in not well understood even today. There are several important factors that control the electron transport in such molecular devices. First one, of course, is the quantization of energy levels associated with the identity of the molecule itself. Second one is the quantum interference effects of electron waves $[8,9,10,11,12,13,14$, associated with the geometry that the molecule adopts within the junction. Third are the different parameters of the Hamiltonian that describe the molecular system, the electronic structure of the molecule and the molecular coupling with the side attached electrodes. The study of structure-conductance relationships is very important for fabrication of efficient molecular devices with specific properties and in a very recent work Ernzerhof et al. [15] have presented a general design principle and performed several model calculations to demonstrate the concept. The knowledge of current fluctuations (of thermal or quantum origin) in molecular devices is also an important issue. Blanter et al. [16 have studied elaborately how the lowest possible noise power of the current fluctuations can be determined in a twoterminal conductor. The steady state current fluctuations, the so-called shot noise, is a consequence of the quantization of charge and it can be used to obtain information on a system which is not available directly through conductance measurements. The noise power of the current fluctuations provides an additional important information about the electron correlation by calculating the Fano factor $(F)$ which directly informs us whether the magnitude of the shot noise achieves the Poisson limit $(F=1)$ or the sub-Poisson $(F<1)$ limit.

Several ab initio methods are used for the calculation of conductance [17, 18, 19, 20, 21, 22, through a molecular bridge system. At the same time the tight-binding model has been extensively studied in the literature and it has also been extended to DFT transport calculations 23, 24. The study of static density functional theory (DFT) [25, 26] within the local-density approximation (LDA) to investigate the electronic transport through nanoscale conductors, like atomic-scale point contacts, has met with nice success. But when this similar theory applies to molecular junctions, theoretical conductances achieve larger values compared to the experimental predictions and these quantitative discrepancies need extensive study in this particular field. In a recent work, Sai et al. 27. have predicted a correction to the conductance using the timedependent current-density functional theory since the dynamical effects give significant contribution in the electron transport, and illustrated some important results with specific examples. Quite similar dynamical effects have also been reported in some other recent papers [28, 29], where authors have abandoned the infinite reservoirs, as originally introduced by Landauer, and considered two large but finite oppositely charged electrodes connected by a nanojunction. Our aim of the present article is to reproduce an analytic approach based on the tight-binding model to characterize the electronic transport properties through some benzene molecules (Fig. 1) and focus our attention on the effects of (a) the quantum interference (b) chemical substituent group (c) the molecule-to-electrode coupling strength and (d) the gate voltage in such transport. Here we utilize a simple parametric approach 30, 31, 32, 33, 34, 35, 36, 37, 38, 39, for these calculations. The model calculations are motivated by the fact that the $a b$ initio theories are computationally too expensive, while, the model calculations by using the tight-binding formulation are computationally very cheap and also provide a worth insight to the problem. In our present study, attention is drawn on the qualitative behavior of the physical quantities rather than the quantitative ones. Not only that, the $a b$ initio theories do not give any 
new qualitative behavior for this particular study in which we concentrate ourselves.

The paper is organized as follow. In Section 2, we describe very briefly about the methodology for the calculation of the transmission probability $(T)$, current $(I)$ and the noise power of current fluctuations $(S)$ through a molecule sandwiched between two metallic electrodes by using the Green's function technique. Section 3 provides the behavior of the conductance as a function of the injecting electron energy, the current and the noise power of its fluctuations as a function of the applied bias voltage for the different molecular wires. In this context we also discuss the effect of the gate voltage on the electron transport through such molecular wires. Finally, we summarize our results in Section 4.

\section{The molecular system and the theoretical formulation}

This section describes the models for the molecular systems and the methodology for the calculation of the transmission probability $(T)$, conductance $(g)$, current $(I)$ and the noise power of its fluctuations $(S)$ through a molecule (schematically represented as in Fig. 1), sandwiched between the two metallic electrodes, by using the Green's function technique.

Figure 1 shows the schematic representation of three different molecular wires in which we concentrate our study. In each wire, the molecule is contacted to the two electrodes (source and drain) and in addition to that each arm of the molecular ring is attached to a voltage gate. The molecular system is described by the tight-binding Hamiltonian. Assuming the gate voltages $V_{a}$ and $V_{b}$ affect only on one atom in each arm of the molecular ring (atoms $a$ and $b$ ), we can write the Hamiltonian for the molecule within the non-interacting picture like,

$$
\begin{aligned}
H_{M} & =\sum_{i}\left(\epsilon_{i 0}+V_{a} \delta_{i a}+V_{b} \delta_{i b}\right) c_{i}^{\dagger} c_{i} \\
& +\sum_{<i j>} t\left(c_{i}^{\dagger} c_{j}+c_{j}^{\dagger} c_{i}\right)
\end{aligned}
$$

where $\epsilon_{i 0}$ 's are the site energies and $t$ is the nearestneighbor hopping strength.

At low voltage and low temperature, the conductance $g$ of the molecule is given by the Landauer conductance formula 40,

$$
g=\frac{2 e^{2}}{h} T
$$

where the transmission probability $T$ is written in this form [40,

$$
T=\operatorname{Tr}\left[\Gamma_{S} G_{M}^{r} \Gamma_{D} G_{M}^{a}\right]
$$

where $G_{M}^{r}\left(G_{M}^{a}\right)$ is the retarded (advanced) Green's function of the molecule, and, $\Gamma_{S}\left(\Gamma_{D}\right)$ describes its coupling to the source (drain). The effective Green's function of the molecule is expressed as,

$$
G_{M}=\left(E-H_{M}-\Sigma_{S}-\Sigma_{D}\right)^{-1}
$$

where $E$ is the energy of the injecting electron and $H_{M}$ is the Hamiltonian of the molecule as de-
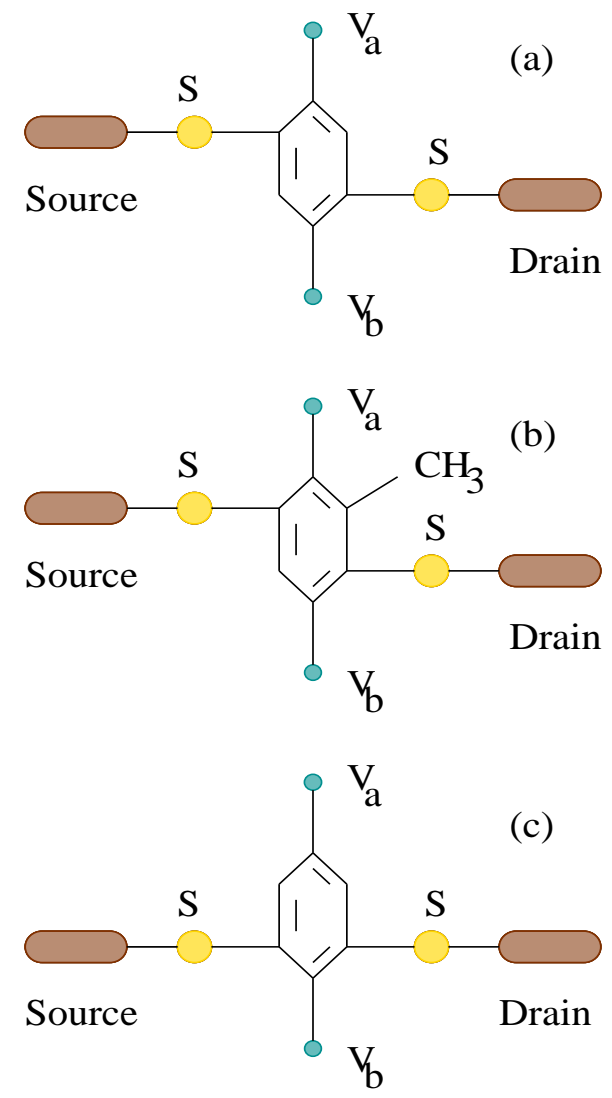

Figure 1: (Color online). Schematic view of three different molecular wires where the benzene molecules are attached to the two electrodes (source and drain) through thiol $(S-H)$ groups in the chemisorption technique. The two gate voltages $V_{a}$ and $V_{b}$ are variable.

scribed in Eq. (1). In Eq. (4), $\Sigma_{S}$ and $\Sigma_{D}$ correspond to the self-energies due to coupling of the molecule to the two electrodes. All the information about the molecule-to-electrode coupling are included into these two self-energies and are described 
through the use of Newns-Anderson chemisorption theory 30, 32.

The current passing across the molecule can be considered as a single electron scattering process between the two reservoirs of charge carriers. The current-voltage relationship can be obtained from the expression [40],

$$
I(V)=\frac{e}{\pi \hbar} \int_{-\infty}^{\infty}\left(f_{S}-f_{D}\right) T(E) d E
$$

where the Fermi distribution function $f_{S(D)}=$ $f\left(E-\mu_{S(D)}\right)$ with the electrochemical potentials $\mu_{S(D)}=E_{F} \pm e V / 2$. For the sake of simplicity, here we assume that the entire voltage is dropped across the molecule-electrode interfaces and this assumption does not greatly affect the qualitative aspects of the $I-V$ characteristics. This assumption is based on the fact that the electric field inside the molecule, especially for short molecules, seems to have a minimal effect on the conductance-voltage characteristics. On the other hand, for quite longer molecules and high bias voltage, the electric field inside the molecule may play a more significant role depending on the internal structure of the molecule [4], yet the effect is too small.

The noise power of the current fluctuations is calculated from the following expression [16],

$$
\begin{aligned}
S= & \frac{2 e^{2}}{\pi \hbar} \int_{-\infty}^{\infty}\left[T(E)\left\{f_{S}\left(1-f_{S}\right)+f_{D}\left(1-f_{D}\right)\right\}\right. \\
& \left.+T(E)\{1-T(E)\}\left(f_{S}-f_{D}\right)^{2}\right] d E
\end{aligned}
$$

where the first two terms of this equation correspond to the equilibrium noise contribution and the last term gives the non-equilibrium or shot noise contribution to the power spectrum. By calculating the noise power of the current fluctuations we can evaluate the Fano factor $F$, which is essential to predict whether the shot noise lies in the Poisson or the sub-Poisson regime, through the relation [16],

$$
F=\frac{S}{2 e I}
$$

For $F=1$, the shot noise achieves the Poisson limit where no electron correlation exists between the charge carriers. On the other hand, for $F<1$, the shot noise reaches the sub-Poisson limit and it provides the information about the electron correlation among the charge carriers.

In this article, we present our results at much low temperature $(5 \mathrm{~K})$, but all the essential features of electron transport are also invariant up to some finite temperature $(\sim 300 \mathrm{~K})$. The reason for such an assumption is that the broadening of the molecular energy levels due to the coupling of the molecule to the electrodes is much larger than that of the thermal broadening. For simplicity, we take the unit $c=e=h=1$ in our present calculations.

\section{Results and discussion}

Here we describe the electron transport characteristics through the three different short molecular wires, schematically represented in Fig. 11 where the molecules are attached to the two electrodes, namely source and drain, respectively. In actual experimental set up these two electrodes are constructed from gold and attached to the molecule via thiol (S-H bond, i.e., sulfur-hydrogen bond) groups in the chemisorption technique, where hydrogen $(\mathrm{H})$ atoms remove and sulfur $(\mathrm{S})$ atoms reside. For the bridge system given in Fig. 1(a), the chemical substituent free benzene molecule is attached to the two electrodes symmetrically, i.e., the upper and the lower arms of the molecular ring have equal length. On the other hand, for the bridge given in Fig. 11(b), a chemical substituent group $\left(\mathrm{CH}_{3}\right)$ is added in one arm of the molecular ring keeping the electrodes at the same positions as the previous one. Here the symmetry is broken by introducing this chemical substituent group which effects the interference conditions. Finally, in the rest molecular bridge, given in Fig. 1)(c), the electrodes are coupled asymmetrically (i.e., the upper and the lower arms of the molecular rings have different lengths) with the chemical substituent free benzene molecule in order to reveal the interference effects on electron transport much more clearly. All these three molecular bridges show various interesting features on the electron transport and we will see that the two gate voltages, $V_{a}$ and $V_{b}$, have significant effect on such transport characteristics.

We will study the behavior of the electron transport through the molecules in two distinct regimes. One is the so-called weak-coupling regime defined as $\tau_{S(D)}<<t$, and the other one is the strongcoupling regime mentioned as $\tau_{S(D)} \sim t$, where $\tau_{S}$ and $\tau_{D}$ are the hopping strengths of the molecule to the source and drain, respectively. In our calculations, the parameters in these two regimes are chosen as $\tau_{S}=\tau_{D}=0.5, t=3$ (weak-coupling) and $\tau_{S}=\tau_{D}=2.5, t=3$ (strong-coupling). The hopping integral in the two electrodes is taken as $v=8$. 

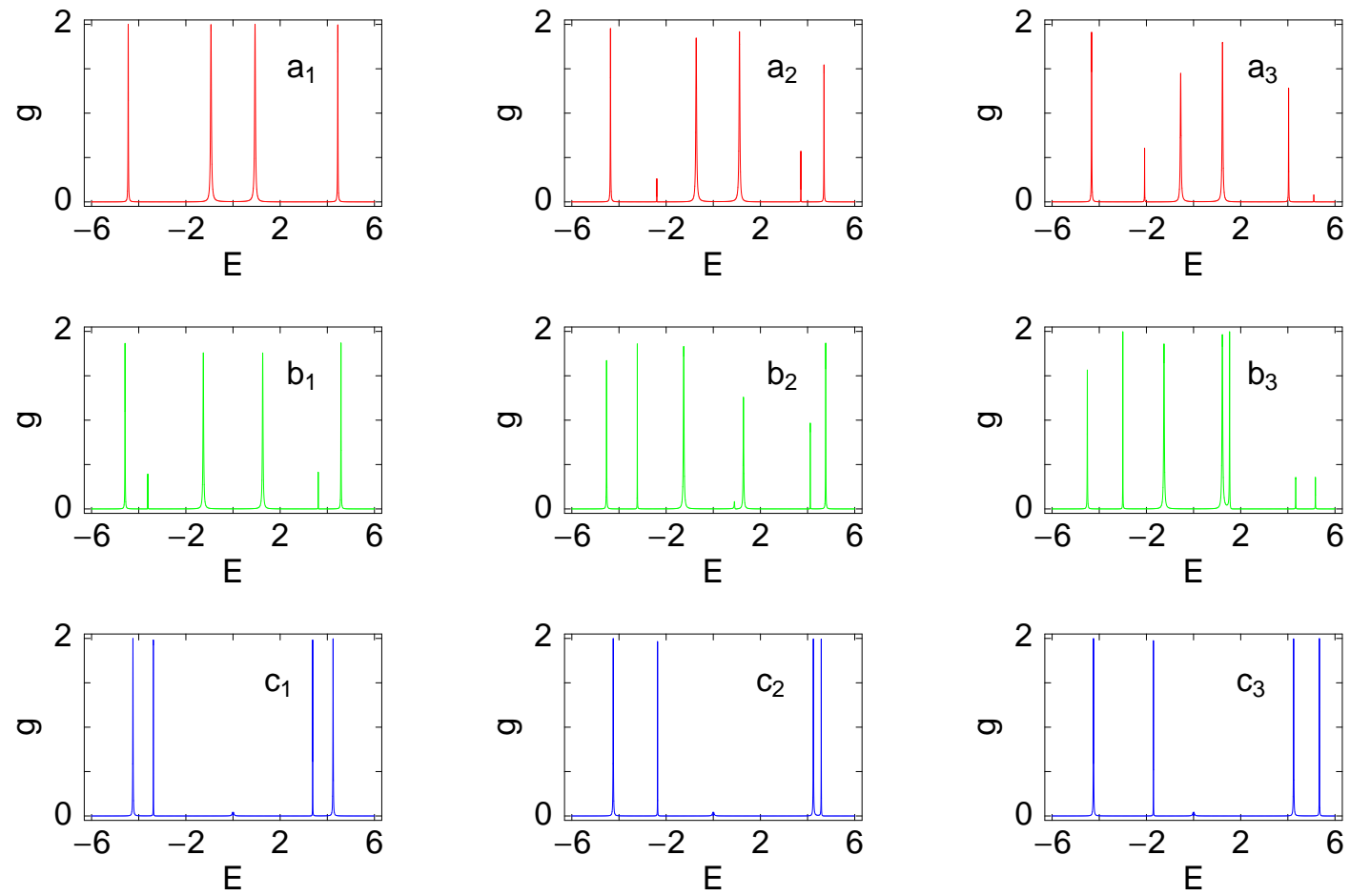

Figure 2: (Color online). Conductance $g$ as a function of the injecting electron energy $E$ in the limit of weak molecular coupling, where the first, second and third rows correspond to the results for the wires given in Figs. 1(a), (b) and (c), respectively. The first, second and third columns represent the results considering the gate voltages as: $V_{a}=0, V_{b}=0 ; V_{a}=3, V_{b}=0$ and $V_{a}=6, V_{b}=0$, respectively.

In Fig. 2 we plot the conductance $g$ as a function of the injecting electron energy $E$ for the three different molecular bridges in the limit of weak molecular coupling, where the first, second and third rows correspond to the results for the bridges given in Figs. 1(a), (b) and (c), respectively. The first column gives the results for the bridges in the absence of any gate voltage $\left(V_{a}=0, V_{b}=0\right)$, while the second and third columns represent the results for the gate voltages $V_{a}=3, V_{b}=0$ and $V_{a}=6, V_{b}=0$, respectively. From all these curves (of Fig. 2) it is observed that the conductance shows very sharp resonant peaks for some particular energies, while for all other energies the conductance vanishes. At the resonant peaks where the conductance $g$ reaches the value 2 , the transmission probability $T$ goes to unity since we get the relation $g=2 T$ from the Landauer conductance formula (see Eq.(2) with $e=h=1$ in our present treatment). The resonant peaks in the conductance spectra are associated with the energy eigenvalues of the single benzene molecules. Therefore, the conductance spectrum manifests itself the electronic structure of the molecule. From the re- sults it is noted that some of the resonant peaks do not achieve the value 2 anymore and also get much reduced values. This behavior can be explained in the following way. The electrons are carried from the source to the drain through the molecules and the electron waves propagating along the two arms of the molecular ring may suffer a relative phase shift between themselves. Accordingly, there might be constructive or destructive interference due to superposition of the electronic wave functions along the various pathways. Therefore, the probability amplitude of the electron across the molecule becomes either large or small. The anti-resonances in the transmission (conductance) spectra are due to the exact cancellation of the transmittances along the two paths. The other key observation is that the positions of the resonant peaks in the conductance spectra get modified with the application of the gate voltages. Thus one can get the on/off state of the molecular bridge for any fixed energy value or applied bias voltage by tuning the external gate voltages, without changing the structure of the molecule itself. This phenomenon is quite significant for 

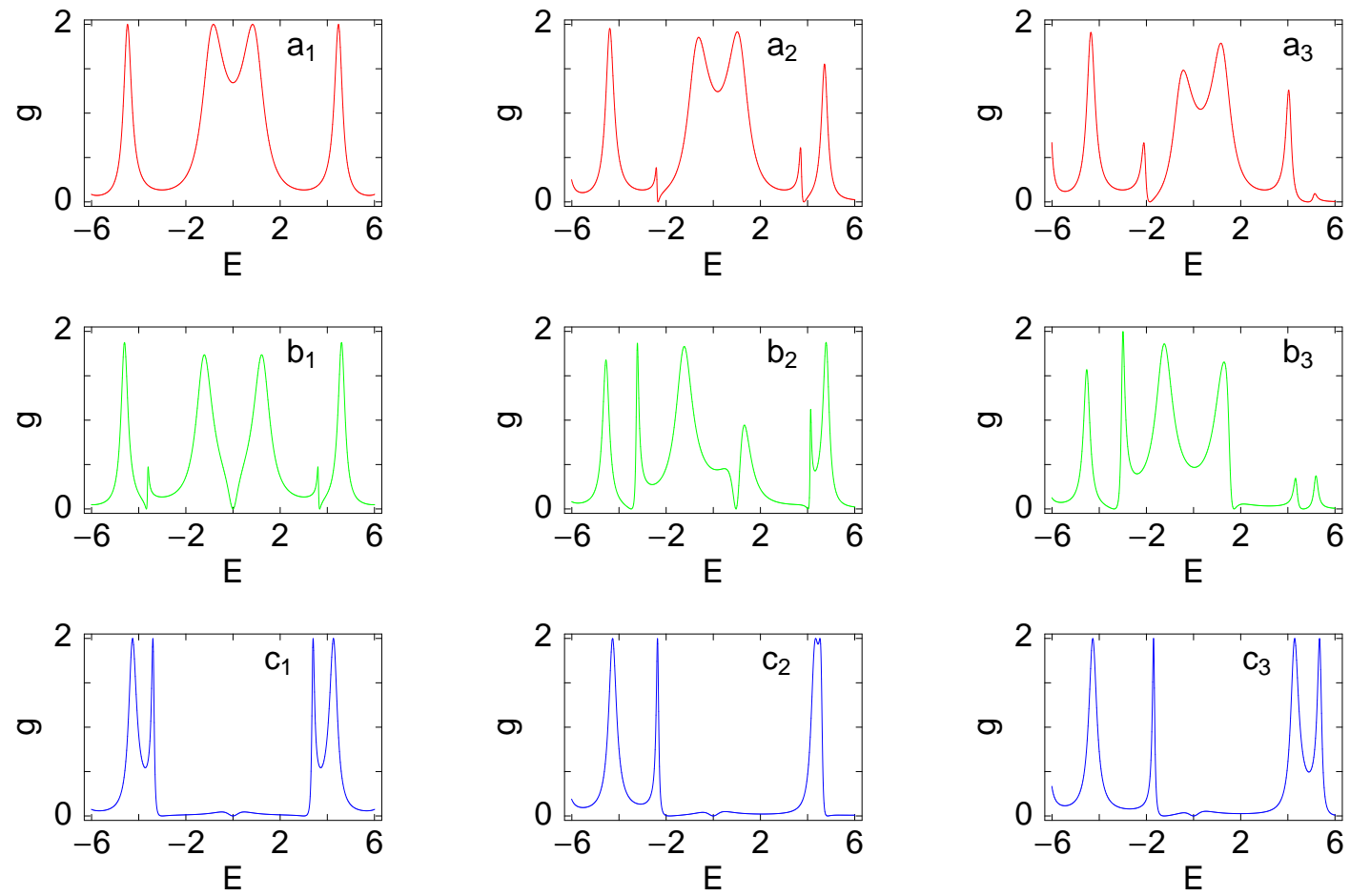

Figure 3: (Color online). Conductance $g$ as a function of the injecting electron energy $E$ in the limit of strong molecular coupling, where the first, second and third rows correspond to the results for the wires given in Figs. 1(a), (b) and (c), respectively. The first, second and third columns represent the results considering the gate voltages as: $V_{a}=0, V_{b}=0 ; V_{a}=3, V_{b}=0$ and $V_{a}=6, V_{b}=0$, respectively.

fabrication of efficient molecular gates or switches. Thus the electron transmission is strongly affected by the quantum interference and can be controlled by the molecule-to-electrode interface structure as well as the external gate voltages.

Now we describe the behavior of the conductance $g$ as a function of the injecting electron energy $E$ for these wires in the limit of strong molecular coupling. The results are shown in Fig. 3, where the figures in the different rows and columns correspond to the same molecular bridges as in Fig. 2 In this strong molecular coupling limit, all the resonant peaks get broadened substantially compared to the weak-coupling case. The strong molecular coupling broadens the molecular energy levels and accordingly, the resonant peaks become wider (contribution comes from the imaginary parts of the two self-energies, $\Sigma_{S}$ and $\Sigma_{D}$ [40]). In the strongcoupling limit, the molecular wires conduct electrons for the wide range of energies compared to the weak-coupling case where fine tuning in the energy scale is necessary to get the electron conduction across the molecules. Thus we can emphasize that the molecular coupling strength has a strong influence on the electron transport. The other important feature appears from the conductance spectra is the existence of the conductance (transmittance) zeros. Such anti-resonances are specific to the interferometric nature of the scattering states and do not occur in the usual one-dimensional scattering problems involving potential barriers. These antiresonant states also appear for such wires in the limit of weak molecular coupling but cannot follow clearly from the curves (plotted in Fig. 2) since the widths of all the resonances are extremely small. It is observed that the positions of the anti-resonant states on the energy scale are independent of the molecule-to-electrode coupling strength. Since the width of these states are very small, they do not give any significant contribution to the current-voltage characteristics. However, the variation of the interference conditions have strong influence on the magnitude of the current flowing through the bridge systems. From the results we can clearly observe the shift of the resonant peaks with the gate voltages (second and third columns of Fig. 3). 


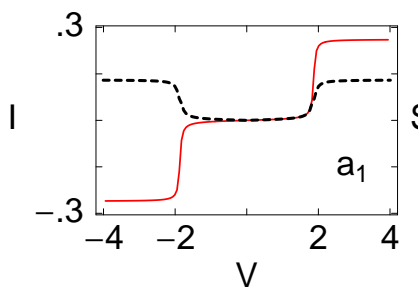

$\mathrm{S} / 2$
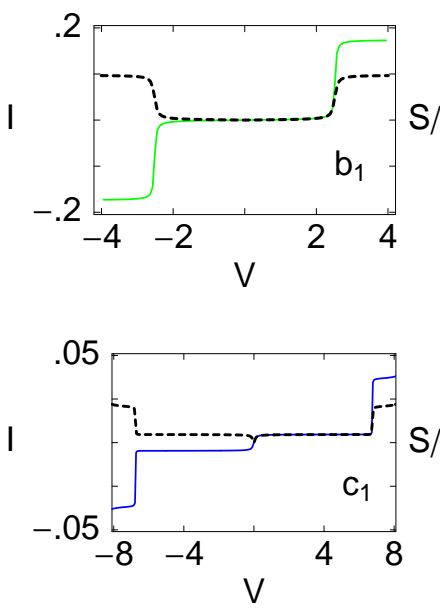

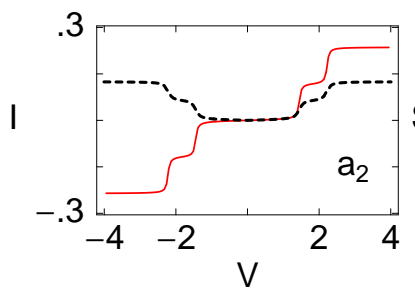

$\mathrm{S} / 2$
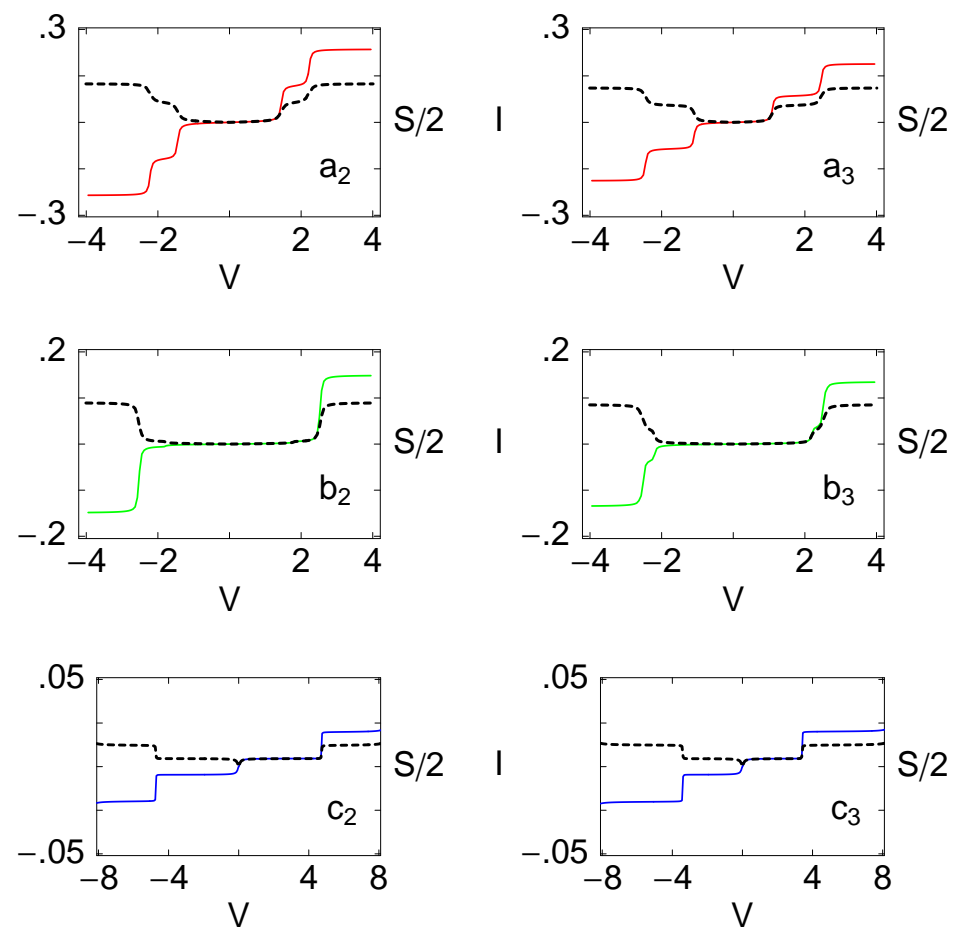

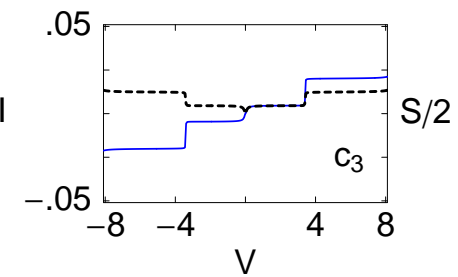

Figure 4: (Color online). Current $I$ and the noise power of its fluctuations $S$ (dotted curve) as a function of the applied bias voltage $V$ in the limit of weak molecular coupling, where the red, green and blue curves correspond to the results for the wires given in Figs. 1(a), (b) and (c), respectively. The first, second and third columns represent the results considering the gate voltages as: $V_{a}=0, V_{b}=0 ; V_{a}=3$, $V_{b}=0$ and $V_{a}=6, V_{b}=0$, respectively.

The scenario of electron transfer through the molecular junction can be understood much more clearly from the current-voltage $(I-V)$ characteristics. Here we shall describe the behavior of the current $I$ and the noise power of current fluctuations $S$ as a function of the applied bias voltage $V$ for these molecular wires, where both the current and the noise power are evaluated by the integration procedure of the transmission function $T$ (see Eqs.(5) and (6)). Figure 4 displays the current $I$ and the noise power of its fluctuations $S$ (dotted curve) of the molecular wires in the limit of weak-coupling, where the red, green and blue curves correspond to the results for the wires given in Figs. 1(a), (b) and (c), respectively. The first column represents the results for the wires in the absence of any gate voltage $\left(V_{a}=0, V_{b}=0\right)$, while, the second and third columns denote the results for the gate voltages $V_{a}=3, V_{b}=0$ and $V_{a}=6, V_{b}=0$, respectively. Several interesting results appear from these curves which we will now discuss one by one. (I) The current $(I)$ shows staircase-like structure with sharp steps as a function of the applied bias voltage $(V)$. This is due to the sharp resonances those appear in the conductance spectra (see Fig. 2) in the weak molecular coupling limit, since the current is evaluated from the integration procedure of the transmission function $T$. With the increase of the applied bias voltage, the electrochemical potentials on the electrodes are gradually shifted and eventually cross one of the molecular energy levels. Accordingly, a current channel is opened up and a jump in the $I-V$ curve appears. (II) The current amplitude through the molecular bridges strongly depends on the geometry of the bridge, which is clearly observed from the curves plotted in the first column of Fig. 4 For the same bias voltage $V$ the current amplitude for the symmetric bridge (Fig. 1(a)) is larger compared to the other two asymmetric bridges (Figs. 1(b) and (c)). This is due to the quantum interference effects of the electron waves traversing through the different arms of the molecular ring. The other important observation is that for a particular bridge the current amplitude can be controlled very nicely 


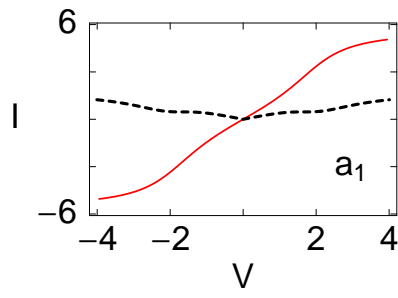

$\mathrm{S} / 2$

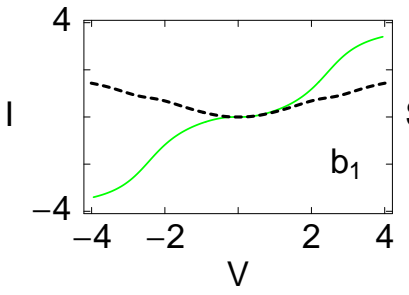

$\mathrm{S} / 2$

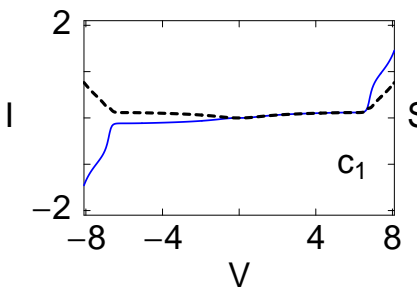

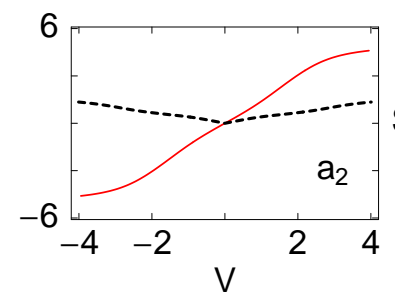

$\mathrm{S} / 2$
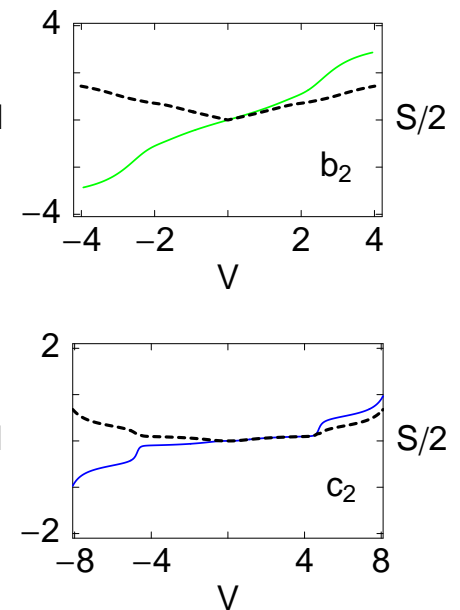
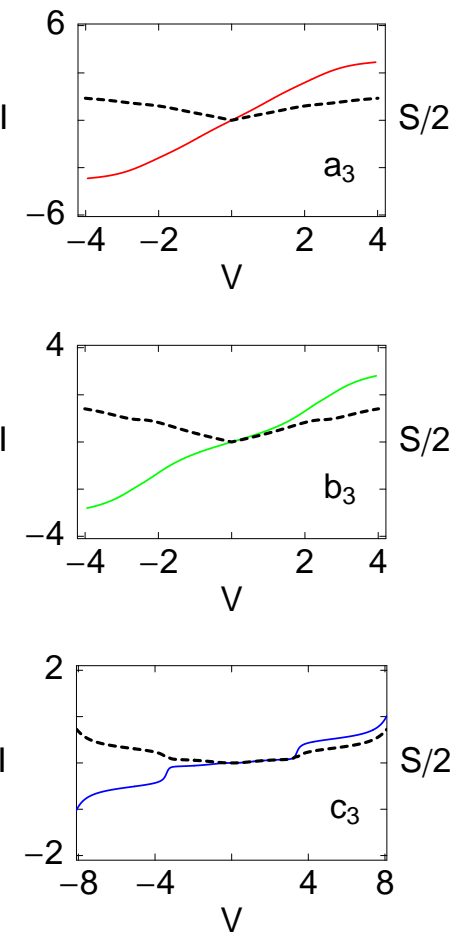

Figure 5: (Color online). Current $I$ and the noise power of its fluctuations $S$ (dotted curve) as a function of the applied bias voltage $V$ in the limit of strong molecular coupling, where the red, green and blue curves correspond to the results for the wires given in Figs. 1(a), (b) and (c), respectively. The first, second and third columns represent the results considering the gate voltages as: $V_{a}=0, V_{b}=0 ; V_{a}=3$, $V_{b}=0$ and $V_{a}=6, V_{b}=0$, respectively.

by applying the two external gate voltages $V_{a}$ and $V_{b}$, which is clarified from the results given in the second and third columns of Fig. 4. The most significant result is that the threshold bias voltage, the voltage where the electron starts conduction through the molecule, can be tuned in a controllable way by these two external gate voltages. Thus we can tune the current amplitude as well as the threshold bias voltage through a molecular bridge, externally, by means of these gate voltages. These results provide key informations for the fabrication of molecular devices. (III) In the determination of the noise power of the current fluctuations $(S)$ (dotted curves of Fig. (4) for these molecular wires it is observed that the shot noise goes from the Poisson limit $(F=1)$ to the sub-Poisson limit $(F<1)$ as long as we cross the first step in the currentvoltage characteristics. This emphasizes that the electrons are correlated after the tunneling process has occurred. Here the electrons are correlated only in the sense that one electron feels the existence of the other according to the Pauli exclusion principle, since we have neglected all other electron-electron interactions in our present treatment.

Now focus our attention on the current and the noise power of its fluctuations for the molecular wires in the limit of strong coupling. The results are shown in Fig. 1 , where the red, green, blue and dotted curves correspond to the same meaning as in Fig. 4. In this strong coupling limit we also get several important features and here summarize them. (I) The current varies almost continuously with the applied bias voltage for all such molecular wires. The key point is that the current amplitudes get enhanced quite significantly compared to the weak molecular coupling limit. This behavior can be clearly understood by noting the areas under the curves in the conductance spectra for this strong molecular coupling limit (see Fig. 3). Thus for a particular bridge system one can enhance the current amplitude by increasing the molecule-toelectrode coupling strength. This is an interesting phenomenon in the study of molecular transport. (II) Like as in the weak-coupling case, here also 
the current amplitude decreases due to the breaking of the symmetry of the molecular bridge (see the second and third rows of Fig. 5). The reason for such behavior is the same as in our previous description. Here also the current amplitudes are controlled by the external gate voltages. (III) Finally, in the study of the noise power of the current fluctuations in this strong coupling case we see that, for the molecular bridge given in Fig. 1(a) there is no such possibility of getting a transition from the Poisson limit $(F=1)$ to the sub-Poisson limit $(F<1)$ since the shot noise already achieves the sub-Poisson limit (see the dotted curves of the first row in Fig. (5), momentarily as we switch on the bias voltage. Therefore, for this particular bridge in this limit of molecular coupling the electron correlation is highly significant. On the other hand, for the other two bridges the shot noise makes a transition from the Poisson limit to the sub-Poisson limit after some critical value of the applied bias voltage (see the dotted curves of the second and third rows in Fig. 5). This critical value of the bias voltage depends on the geometry of the molecular bridge as well as the external gate voltages for the fixed molecular coupling strength.

\section{Concluding remarks}

To summarize, we have studied the electron transport properties through some molecular wires, based on the tight-binding model, by using the Green's function formalism. The transport properties through the wires are significantly affected by the several factors like, (a) quantum interference of the electron waves traversing through the different arms of the molecular ring, (b) chemical substituent group, (c) molecular coupling to the electrodes and (d) the external gate voltages. All the characteristic features described here provide several key ideas for fabrication of efficient molecular devices.

For the weak molecular coupling limit the conductance shows fine resonant peaks (Fig. 2) for some particular energy values, while, for all other energies it drops to zero which are in fact the signature of the electronic structure of the molecules. On the other hand, in the limit of strong molecular coupling the width of these resonant peaks get broadened substantially (Fig. 3). This is due to the broadening of the molecular energy levels, where the contribution comes from the imaginary parts of the self energies $\Sigma_{S}$ and $\Sigma_{D}$ [40]. When the molecular symmetry is broken, more anti-resonant peaks appear in the conductance spectra and their posi- tions are independent of the molecule-to-electrode coupling strength.

The scenario of the electron transfer through the molecular bridges can be visible much more clearly by studying the current-voltage characteristics. Current shows the staircase-like behavior with sharp steps (colored curves in Fig. 4) in the limit of weak molecular coupling, while, it gets a continuous variation (colored curves in Fig. 5) as we increase the coupling strength and achieves much larger amplitude. The current amplitude can also be tuned by applying the external gate voltages (second and third columns of Figs. 4 and 5).

Finally, in the determination of the noise power of the current fluctuations we have seen that whether the shot noise lies in the Poisson limit $(F=1)$ or the sub-Poisson limit $(F<1)$ strongly depends on the geometry of the molecular bridge as well as on the molecular coupling strength (dotted curves in Figs. 4 and 50.

Throughout our discussion, we have used several approximations by neglecting the effects of the electron-electron interaction, all the inelastic scattering processes, the Schottky effect, the static Stark effect, etc. More studies are expected to take into account all these approximations for our further investigations.

\section{References}

[1] A. Aviram and M. Ratner, Chem. Phys. Lett. 29, 277 (1974).

[2] M. A. Reed, C. Zhou, C. J. Muller, T. P. Burgin and J. M. Tour, Science 278, 252 (1997).

[3] C. P. Collier, E. W. Wong, M. Belohradsky, F. M. Raymo, J. F. Stoddart, P. J. Kuekes, R. S. Williams and J. R. Heath, Science 285, 391 (1999).

[4] R. M. Metzger, J. Mater. Chem. 10, 55 (2000).

[5] T. Rueckes, K. Kim, E. Joselevich, G. Y. Tseng, C. L. Cheung and C. M. Lieber, Science 289, 94 (2000).

[6] C. P. Collier, G. Mattersteig, E. W. Wong, Y. Luo, K. Beverly, J. Sampaio, F. M. Raymo, J. F. Stoddart and J. R. Heath, Science 289, 1172 (2000).

[7] C. Joachim, J. K. Gimzewski and A. Aviram, Nature 408, 541 (2000). 
[8] M. Magoga and C. Joachim, Phys. Rev. B 59, 16011 (1999).

[9] J.-P. Launay and C. D. Coudret, in: A. Aviram and M. A. Ratner (Eds.), Molecular Electron$i c s$, New York Academy of Sciences, New York, (1998).

[10] R. Baer and D. Neuhauser, Chem. Phys. 281, 353 (2002).

[11] R. Baer and D. Neuhauser, J. Am. Chem. Soc. 124, 4200 (2002).

[12] D. Walter, D. Neuhauser and R. Baer, Chem. Phys. 299, 139 (2004).

[13] R. H. Goldsmith, M. R. Wasielewski and M. A. Ratner, J. Phys. Chem. B 110, 20258 (2006).

[14] M. Ernzerhof, H. Bahmann, F. Goyer, M. Zhuang and P. Rocheleau, J. Chem. Theory Comput. 2, 1291 (2006).

[15] M. Ernzerhof, M. Zhuang and P. Rocheleau, J. Chem. Phys. 123, 134704 (2005).

[16] Y. M. Blanter and M. Büttiker, Phys. Rep. 336, 1 (2000).

[17] S. N. Yaliraki, A. E. Roitberg, C. Gonzalez, V. Mujica and M. A. Ratner, J. Chem. Phys. 111, 6997 (1999).

[18] M. Di Ventra, S. T. Pantelides and N. D. Lang, Phys. Rev. Lett. 84, 979 (2000).

[19] Y. Xue, S. Datta and M. A. Ratner, J. Chem. Phys. 115, 4292 (2001).

[20] J. Taylor, H. Gou and J. Wang, Phys. Rev. B 63, 245407 (2001).

[21] P. A. Derosa and J. M. Seminario, J. Phys. Chem. B 105, 471 (2001).

[22] P. S. Damle, A. W. Ghosh and S. Datta, Phys. Rev. B 64, R201403 (2001).

[23] M. Elstner, D. Porezag, G. Jungnickel, J. Elsner, M. Haugk, T. Frauenheim, S. Suhai and G. Seifert, Phys. Rev. B 58, 7260 (1998).

[24] T. Frauenheim, G. Seifert, M. Elstner, T. Neihaus, C. Köhler, M. Amkreutz, M. Sternberg, Z. Hajnal, A. D. Carlo and S. Suhai, J. Phys.: Condens. Matter 14, 3015 (2002).

[25] P. Hohenberg and W. Kohn, Phys. Rev. 136, B864 (1964).
[26] W. Kohn and L. J. Sham, Phys. Rev. 140, A1133 (1965).

[27] N. Sai, M. Zwolak, G. Vignale and M. D. Ventra, Phys. Rev. Lett. 94, 186810 (2005).

[28] N. Bushong, N. Sai and M. D. Ventra, Nano Lett. 5, 2569 (2005).

[29] M. D. Ventra and T. N. Todorov, J. Phys.: Condens. Matter 16, 8025 (2004).

[30] V. Mujica, M. Kemp and M. A. Ratner, J. Chem. Phys. 101, 6849 (1994).

[31] S. K. Maiti, Int. J. Nanosci. 7, 51 (2008).

[32] V. Mujica, M. Kemp, A. E. Roitberg and M. A. Ratner, J. Chem. Phys. 104, 7296 (1996).

[33] S. K. Maiti, J. Nanosci. Nanotechnol. 8, 4096 (2008).

[34] M. P. Samanta, W. Tian, S. Datta, J. I. Henderson and C. P. Kubiak, Phys. Rev. B 53, R7626 (1996).

[35] S. K. Maiti, Solid State Commun. 149, 1623 (2009).

[36] M. Hjort and S. Staftröm, Phys. Rev. B 62, 5245 (2000).

[37] S. K. Maiti, Solid State Commun. 149, 1684 (2009).

[38] K. Walczak, Phys. Stat. Sol. (b) 241, 2555 (2004).

[39] K. Walczak, Cent. Eur. J. Chem. 2, 524 (2004).

[40] S. Datta, Electronic transport in mesoscopic systems, Cambridge University Press, Cambridge (1997).

[41] W. Tian, S. Datta, S. Hong, R. Reifenberger, J. I. Henderson and C. I. Kubiak, J. Chem. Phys. 109, 2874 (1998). 